\title{
Innovare i sistemi di controllo e di governance per gestire il cambiamento
}

\author{
Aldo Pavan ${ }^{*}$, Giuseppe D’Onza ${ }^{* *}$
}

Gli andamenti sfavorevoli che da alcuni anni stanno caratterizzando lo scenario economico dei cosiddetti Paesi a "Economia Avanzata" hanno indotto molte aziende a ripensare le caratteristiche del loro modello gestionale e organizzativo, con l'obiettivo di salvaguardare le performance competitive, economico-finanziarie e sociali. In un contesto in cui numerose organizzazioni sono chiamate a ripensare se stesse per rispondere ai cambiamenti talvolta repentini dell'ambiente, i sistemi di controllo interno e di corporate governance possono offrire un importante contributo tanto in fase di pianificazione del change management, per identificare le variabili su cui intervenire, quanto durante il percorso di attuazione del cambiamento, per vincere alcune possibili resistenze. I contributi riportati in questo numero della rivista offrono delle indicazioni al riguardo, tutte basate sull'interazione metodologica tra le dimensioni teorica ed empirica, interessanti in quanto presentano delle vie di innovazione nei sistemi di controllo e di governance che possono rendere entrambe le variabili più funzionali alle esigenze attuali delle organizzazioni.

I manager si aspettano di ricevere dai controlli interni informazioni utili e tempestive sia per individuare le attività a basso valore aggiunto da eliminare, ridisegnare o riprogettare, sia per identificare i processi che possono presentare delle potenzialità inespresse da valorizzare. Tali informazioni sono divenute sempre più importanti in un contesto in cui le esigenze di riconfigurare la catena del valore si manifestano con maggiore frequenza. Laddove i sistemi di controllo, per carenze strutturali o di funzionamento, non riescono a soddisfare tali attese divengono anch'essi attività a basso valore aggiunto, di scarsa valenza per le decisioni strategiche e operative, destinati, inevitabilmente, a un ruolo sempre più marginale nel quadro dei sistemi manageriali.

\footnotetext{
*Università di Cagliari, apavan@unica.it.

** Università di Pisa, gdonza@ec.unipi.it.
} 
I primi tre articoli riportati nel volume si muovono lungo questa direzione che potremmo definire di miglioramento dell'efficacia dei sistemi di controllo, dove per efficacia s'intende la capacità degli stessi di soddisfare $i$ fabbisogni conoscitivi del management ai vari livelli organizzativi. Nel contributo di Beretta e Bozzolan, il miglioramento consiste nell'integrazione tra key performance e key risk indicator per arricchire $\mathrm{i}$ sistemi di controllo diagnostico dell'azienda, con indicatori predittivi utili per monitorare l'andamento dei rischi insiti nei vari processi che caratterizzano il modello di business dell'azienda.

L'importanza di inserire nei sistemi di valutazione delle performance la dimensione del rischio aziendale è evidenziata anche nel lavoro di Galeotti, Ferri e De Luca che propone un sistema integrato di indicatori tali da permettere al top management di valutare il posizionamento competitivo dell'azienda nei mercati finanziari. Gli indicatori di performance proposti riguardano i rischi aziendali e altre tre variabili (le prospettive economiche della gestione, la differenziazione dell'offerta finanziaria, la qualità della gestione) che devono essere monitorate per valutare la capacità dell'azienda di risultare attrattiva sui mercati dei capitali e per impostare le strategie finanziarie.

Nell'articolo di Bracci l'intervento di miglioramento dei controlli viene presentato come attività preliminare rispetto alla fase di ridisegno dei processi secondo i principi del lean office. Per essere efficacemente attuato il ridisegno dei processi richiede infatti la disponibilità di metriche di valutazione che permettono all'analista di individuare le attività in cui si annidano sprechi e inefficienze e di identificare, contemporaneamente, i driver fondamentali da cui dipende la qualità degli output per il cliente finale.

Questa esigenza di monitorare le variabili critiche per l'efficienza dei processi e per la qualità degli output per i clienti finali costituisce un elemento trasversale ai diversi settori di attività. Tale fabbisogno conoscitivo viene evidenziato, infatti, anche nel contributo di Gori e Fissi che riguarda la governance dei servizi pubblici locali. In questo settore i cambiamenti normativi, i vincoli di finanza pubblica e altri fattori richiederebbero lo sviluppo di modelli aziendali in cui il controllo tramite "rappresentanza", svolto grazie agli amministratori nominati dagli enti locali, dovrebbe essere integrato da un sistema di reporting integrato che permetta di monitorare l'efficacia e la qualità dei servizi erogati. Tra questi l'analisi della soddisfazione dei clienti non dovrebbe essere considerato meno importante rispetto al monitoraggio dei costi sostenuti per prestare il servizio. Gli autori evidenziano tuttavia come, dalla ricerca condotta, emerge che in molti casi i sistemi di reporting non contengono le informazioni richieste per monitora- 
re l'efficacia e la qualità dei servizi, limitando così la capacità dei Comuni di misurare il valore socio-economico delle prestazioni erogate e riducendo, in molti casi, il controllo analogo a un meccanismo puramente formale.

La difficile situazione economica che sta caratterizzando il nostro Paese fa da cornice al lavoro di Gaia che ha analizzato uno dei temi più rilevanti e dibattuti della governance: la remunerazione dell'amministratore delegato. L'indagine condotta evidenzia che durante i periodi di crisi gli executive director, grazie a un maggior peso della remunerazione fissa, finiscono per espropriare ricchezza a danno degli azionisti e, in particolare, di quelli di minoranza. Da ciò l'esigenza di ripensare le politiche retributive per evitare il crearsi di uno scollamento tra la remunerazione dell'amministratore delegato e le performance aziendali, puntando anche a una maggior trasparenza informativa per rafforzare i meccanismi di controllo nell'area della governance. 